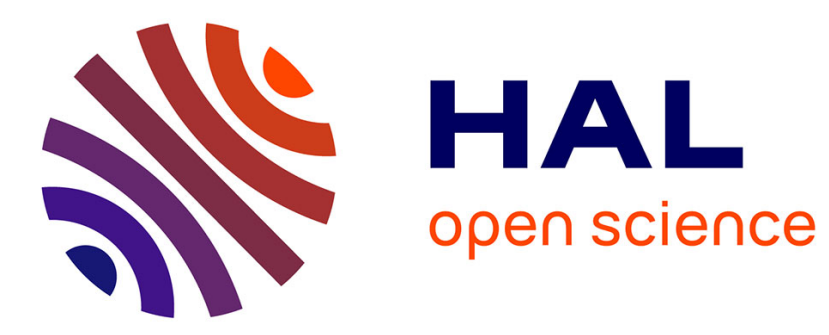

\title{
Numerical and experimental investigation of mucociliary clearance breakdown in cystic fibrosis
}

\author{
Robin Chatelin, Dominique Anne-Archard, Marlene Murris-Espin, Marc \\ Thiriet, Philippe Poncet
}

\section{- To cite this version:}

Robin Chatelin, Dominique Anne-Archard, Marlene Murris-Espin, Marc Thiriet, Philippe Poncet. Numerical and experimental investigation of mucociliary clearance breakdown in cystic fibrosis. Journal of Biomechanics, 2017, 53, pp.56-63. 10.1016/j.jbiomech.2016.12.026 . hal-01476172v2

\section{HAL Id: hal-01476172 \\ https://hal.inria.fr/hal-01476172v2}

Submitted on 2 Nov 2020

HAL is a multi-disciplinary open access archive for the deposit and dissemination of scientific research documents, whether they are published or not. The documents may come from teaching and research institutions in France or abroad, or from public or private research centers.
L'archive ouverte pluridisciplinaire HAL, est destinée au dépôt et à la diffusion de documents scientifiques de niveau recherche, publiés ou non, émanant des établissements d'enseignement et de recherche français ou étrangers, des laboratoires publics ou privés. 


\title{
Numerical and experimental investigation of mucociliary clearance breakdown in cystic fibrosis
}

\author{
Robin Chatelin ${ }^{1}$, Dominique Anne-Archard ${ }^{2}$, Marlène Murris-Espin ${ }^{3}$, Marc Thiriet $^{4}$, Philippe Poncet ${ }^{5}$ \\ ${ }^{1}$ Université de Lyon, ENI Saint Etienne, LTDS, UMR CNRS 5513, 58 rue Jean Parot, 42023 Saint-Etienne Cedex 2, France \\ ${ }^{2}$ Institut de Mécanique des Fluides de Toulouse (IMFT) - Université de Toulouse, CNRS-INPT-UPS, Allée Camille Soula, Toulouse, France \\ ${ }^{3}$ Service de pneumologie et allergologie, CHU de Toulouse, Hôpital Larrey, \\ 24 chemin de Pouvourville, TSA 30030, F-31059 Toulouse Cedex 9, France \\ ${ }^{4}$ CNRS, Lab. JL. Lions, UMR CNRS 7598, Univ. P. \& M. Curie, 75252 Paris, France \\ ${ }^{5}$ University Pau \& Pays Adour, LMAP, UMR CNRS 5142, IPRA, avenue de l'Université, F-64013 Pau, France
}

\begin{abstract}
The human tracheobronchial tree surface is covered with mucus. A healthy mucus is an heterogeneous material flowing toward the esophagus and a major defense actor against local pathogen proliferation and pollutant deposition. An alteration of mucus or its environment such as in cystic fibrosis dramatically impacts the mucociliary clearance. In the present study, we investigate the mechanical organization and the physics of such mucus in human lungs by means of a joint experimental and numerical work. In particular, we focus on the influence of the shear-thinning mucus mobilized by a ciliated epithelium for mucociliary clearance. The proposed robust numerical method is able to manage variations of more than 5 orders of magnitude in the shear rate and viscosity. It leads to a cartography that allows to discuss major issues on defective mucociliary clearance in cystic fibrosis. Furthermore, the computational rheological analysis based on measurements shows that cystic fibrosis shear-thinning mucus tends to aggregate in regions of lower clearance. Yet, a rarefaction of periciliary fluid has a greater impact than the mucus shear-thinning effects.
\end{abstract}

Keywords: Computational biology, Cystic fibrosis, Mucociliary clearance, Modeling, Rheology, Generalized Stokes equations, Penalization, Grid-particle methods.

\section{Introduction}

The human respiratory tract (nose, pharynx, larynx, trachea, bronchi, bronchioles, and alveoli) and its related cavities and conduits (sinuses and Eustachian tubes) has a wetted surface covered with mucus flowing toward the esophagus. Human conducting airways are lined with a pseudostratified, secretory, and ciliated epithelium (with ciliated, secretory, and basal cells) coating submucosal glands as well as cartilaginous structures in some segments of the respiratory tract such as partial or complete rings in the proximal part of the tracheo-bronchial tree.

An efficient mucociliary transport requires an appropriate mucus composition for optimal clearance, adequate production of periciliary fluid and number of functioning ciliated cells, and coordinated motion of cilia for mucus propulsion, as well as respiratory epithelium integrity over long distances. The mucociliary clearance protects the airway epithelium against inhaled pathogens and noxious entrapped micro- and nanoparticles. The mucociliary clearance also influences drug delivery to airways, drug absorption at a given station of the respiratory tract depending on the local rate of mucus transport.

\footnotetext{
${ }^{1}$ Corresponding author: philippe.poncet@univ-pau.fr
}

The present study focuses on impaired mucociliary transport in cystic fibrosis. Cystic fibrosis (CF) is one of the most common life-shortening genetic diseases. It is observed on 1 in every 2500 infants in Europe and North America. It is most common among Caucasian population ( 1 in 22 individuals is a heterozygous carrier). The heterozygote frequency reaches $4 \%$ of the population of Western world. The prevalence of cystic fibrosis per 10000 inhabitants in the United States of America is 0.797 and is on average 0.737 in the 27 European Union countries (with a remarkable 2.98 in the Republic of Ireland) [23].

Defective mucus composition alters mucociliary transport. Impaired chloride transport in cystic fibrosis prevents normal hydration of mucus, leading to highly viscous accumulations. Cystic fibrosis airways are characterized by airway surface liquid volume depletion and mucus accumulation, thereby hampering airway clearance of inhaled pollutants and pathogens [17].

Our numerical and rheological investigations aim at providing an independent and self-consistent analysis of mucociliary clearance altered by cystic fibrosis allowing full reproducibility. Questions needed to be answered encompass the role on mucus viscosity of the disease itself, associated infection, and influence of the periciliary fluid (PCL). Current models assume two Newtonian layers of different viscosities or a single complex 
fluid [39], or two layers with a constant visco-elastic parameter superposed to a spatially varying diffusion [30]. In the present article, a rheological hydration/maturation law incorporates the mucin transfer through a Newtonian periciliary fluid and mucin meshwork formation in a non-Newtonian mucus layer (ML) with smooth and sharp transitions.

Many rheological laws have been identified or used in the literature, from shear-thinning to viscoelastic models but nonshear-thinning (Maxwell [40, 56], Oldroyd B model [44, 43, 30]), viscoplastic (Bingham [66], investigation of 2D mucus without cilia, Carreau [41] with spatially constant parameters, and Herschel-Bulkley [37] constitutive laws). Mucus rheology depends on the context (mucus conservation, illness severity, treatment effect, mucus source, sampling mode, etc.). As mentioned by Besseris and Yeates [5]: "no constitutive law ever was proposed for this material because it was never clearly classified either as a chemically or physically linked gel".

The major issue of this study is to quantify the mucociliary clearance and to investigate the influence of the highly shearthinning nature of mucus. To do that, a state-of-the-art solver is used to compute the variable-viscosity Stokes problem in a mobile domain due to beating cilia [8]. This model deals with noncartilaginous ciliated walls of the tracheobronchial tree, where the ciliated surface is assumed to be periodically flat.

This article is structured as follows. Firstly, the experimental method is described from samples collection to measurement validation. Secondly, we introduce the mathematical model for flow and maturation which enables the transition from the Newtonian PCL to non-Newtonian ML. The numerical method solving efficiently this heterogeneous fluid dynamics around beating cilia is then described. It involves only standard elliptic problems and avoid matrix assembly, thereby leading to a low storage method [9]. This is then applied to a wide range of rheological parameters, which gives a cartography of mucociliary efficiency. This allows comparison of our data with literature data.

In addition to the agreement between experimental observations and numerical parametric studies, the situation of healthy and pathological mucus is achieved on a map. A collection of these maps is finally provided in order to quantify the impact of PCL reduction on mucociliary clearance.

\section{Rheology measurements and experimental setup}

The main and mandatory property for numerical simulations of shear-thinning fluids is the viscosity function $\eta(\dot{\gamma})$ with respect to the shear-rate $\dot{\gamma}$, the full contraction of the symmetric part of the gradient of the fluid velocity $u$ :

$$
\dot{\gamma}=(2 D(u): D(u))^{1 / 2}
$$

where $D(u)=\left(\nabla u+\nabla u^{T}\right) / 2$. Two types of equipments are used to assess this parameter: classical rheometers with a cone and plate or parallel plates geometry $[42,54,58,61]$, and magnetic microrheometers which only allow small amplitude oscillatory motions [34, 49, 67].
Experiments were carried out on a Mars III rheometer (Thermo Sc.) equipped with a cone and plate geometry (angle $1^{\circ}$, diameter $35 \mathrm{~mm}$ ) which requires a small volume of fluid $(0.2 \mathrm{ml})$. Measurements were conducted at the body temperature $\left(37^{\circ} \mathrm{C}\right)$. The lower plate Peltier system for temperature regulation was completed by a home-made sample hood with a specific temperature regulation and wet sponges to saturate the measuring chamber and to prevent samples evaporation.

The rheological characterization consists of dynamic measurements (small amplitude oscillations) with determination of the linear domain followed by measurement of the storage $G^{\prime}$ and loss modulus $G^{\prime \prime}$ as functions of the oscillation frequency $\omega$. $G^{\prime}(\omega)$ and $G^{\prime \prime}(\omega)$ are respectively the elastic (in-phase) and viscous (out-of-phase) components of the complex modulus $G^{*}=G^{\prime}+i G^{\prime \prime}$ which is the ratio between shear stress and strain during oscillation measurements. The viscosity $\eta(\dot{\gamma})$ was then determined by a steady shear flow test for shear rates ranging from $10^{-3} s^{-1}$ to $10^{3} s^{-1}$. A special attention was paid to reach a steady state regime for low shear rate values.

\subsection{Methodology validation}

Mucus was sampled during consultation (in stabilized CF patients as well as in a patient with bronchiectasis without $\mathrm{CF}$ ) or hospitalization (both decompensated or stabilized posttherapeutic states). Samples were frozen before transport and processing. Comparison between fresh and frozen mucus (freezing duration 21 and 30 days) were carried out on three samples and confirmed that the freezing does not alter the rheological propoerties as it was already observed by Sanders et al. [50] and Rubin et al. [49].

Thixotropy is sometimes mentioned in mucus studies and related measurements [46]. For a given patient, when the mucus sample volume was sufficient, shear viscosity was successively measured on the same and on different samples. The dispersion observed using these two series of measures was similar, that is, thixotropy was comparable to the natural dispersion arising from heterogeneity of the sample. The weak thixotropy was already observed in viscosity measurements for increasing and decreasing shear rate [65]. In addition, no phase separation was observed during defrosting and during measurement.

Fifteen mucus samples were analyzed and among them two were rejected. They were visually too heterogeneous and gave rise to a large dispersion in viscosity and modulus values.

\subsection{Rheological results}

A shear-thinning behavior is observed in the entire shear rate range. The storage modulus $G^{\prime}(\omega)$ and the viscous modulus $G^{\prime \prime}(\omega)$ clearly display the viscoelastic character of mucus. These curves cross each other at a frequency $\omega_{c}$. The characteristic time defined from $\omega_{c}$ is of the order $10^{2} \mathrm{~s}$.

A power law model $\eta=K \dot{\gamma}^{N-1}$ was fitted on the shear rate range $\left[0.01-100 s^{-1}\right.$, which is representative of shear rate involved in the studied flow. Values for the power-law index $N$ and the consistency $K$ are obtained by mean-square log-fitting of measures (see Fig. 1 and Table 1). Three examples of steadystate shear viscosity curves for different mucus from two to 


\begin{tabular}{|c|c|c|c|}
\hline Alias & $\mathrm{N}$ & K & Source \\
\hline CFL1 & 0.408 & 0.544 & [54] \\
\hline CFL2 & 0.182 & 1.397 & [61] \\
\hline CFL3 & 0.166 & 2.245 & [61] \\
\hline CFL4 & 0.163 & 2.423 & [61] \\
\hline CFL5 & 0.17 & 7.34 & [15] \\
\hline HL6 & 0.19 & 14.1 & [34] \\
\hline HL7 & 0.195 & 15.49 & [34] \\
\hline HL8 & 0.215 & 13.18 & [67] \\
\hline HL9 & 0.482 & 0.355 & [54] \\
\hline CFM1 & 0.20 & 5.80 & \\
\hline CFM2 & 0.39 & 0.45 & \\
\hline CFM3 & 0.32 & 1.24 & \\
\hline CFM4 & 0.39 & 1.60 & \\
\hline CFM5 & 0.30 & 2.37 & \\
\hline CFM6 & 0.23 & 4.22 & \\
\hline CFM7 & 0.21 & 3.10 & \\
\hline CFM8 & 0.23 & 3.53 & \\
\hline CFM9 & 0.37 & 0.51 & \\
\hline CFM10 & 0.36 & 2.17 & \\
\hline CFM11 & 0.22 & 4.75 & \\
\hline BM12 & 0.40 & 1.43 & \\
\hline
\end{tabular}

Table 1: Rheological parameters $(K, N)$ from collected samples (CFM1-CFM11 standing for Cystic Fibrosis, and BM12 standing for Bronchiectasis) and the literature (CFL1-CFL5 and HL6-HL9 from the complex modulus $\mathrm{G}^{*}$ ). Aliases CFL and HL stand for "Cystic Fibrosis from Literature" and "Healthy cases from Literature".

three measurements, each on different mucus samples are displayed in Fig. 1. These curves illustrate both the usual intraand inter-subject variability of any biological material.

Few literature data on human airway mucus are available for steady state viscosity, whereas numerous data exist for complex viscosity $\eta^{*}=G^{*} / \omega$, giving

$$
\left|\eta^{*}\right|=\left|G^{*}\right| / \omega=\sqrt{G^{\prime}(\omega)^{2}+G^{\prime \prime}(\omega)^{2}} / \omega
$$

The $\left|\eta^{*}\right|$ modulus is considered as an admissible estimation of viscosity when shear viscosity is not obtainable (Cox-Merz rule [6]), and this was largely used. Nevertheless, all authors recognize a strong shear-thinning behavior. This is illustrated by the power law parameters extracted from literature data on $G^{\prime}$ and $G^{\prime \prime}$ and displayed on Table 1, which constitutes a comparison basis for the shear viscosity measured in this study. Whereas some authors $[42,15,58]$ observed a small Newtonian plateau at low shear rates $\left(0.1-1.0 s^{-1}\right)$, others [65, 46, 47] did not monitor any trend to a plateau although exploring shear rate from $0.01 s^{-1}$. Our own experience does not reveal any plateau even for shear rate values of $10^{-3} s^{-1}$. This behavior is generally linked to viscoplasticity, but the $\tau(\dot{\gamma})$ curves obtained on the tested samples do not display any tendency to a yield stress. So, from our measurements, it appears that, if a yield stress exists, it is likely very low (typically $<0.01 P a$ ).

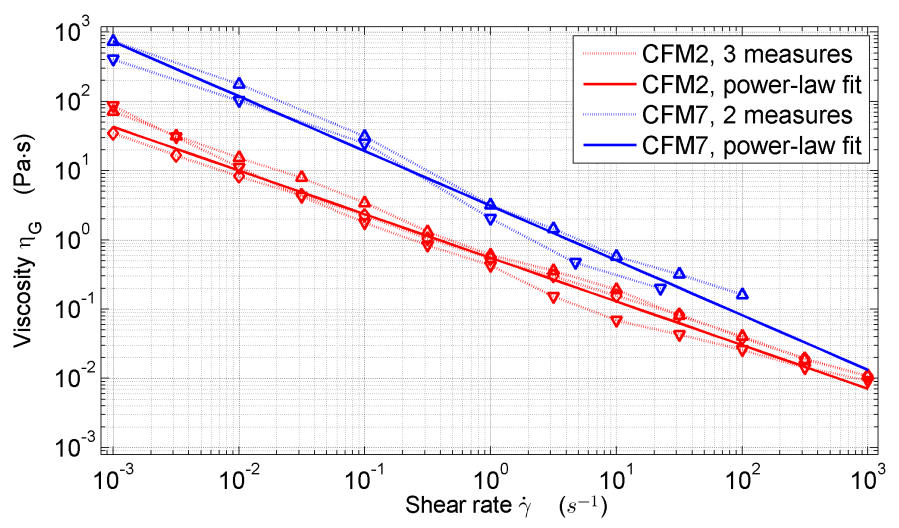

Figure 1: Curves from CFM2 and CFM7 measurements from Table 1 with their best-fitting power-law on $\dot{\gamma} \in\left[10^{-2}, 10^{2}\right]$.

The power-law index $N$ values reported in Table 1 , which derive from rheograms $\eta(\dot{\gamma})$ ranges from 0.2 to 0.4 ; they are similar to literature data $[47,58]$. Values of consistency $K$ and viscosity are also similar. All viscosity curves are limited by two envelope curves defined by $\left(K_{\min }=0.45, N_{\min }=0.39\right)$ and $\left(K_{\max }=5.8, N_{\max }=0.20\right)$. The large shear rate range explored in this study gives an appropriate description of viscosity for the mucus film displacement.

\section{Modeling of mucus maturation}

This section first presents the airway surface liquid structure, composed of mucus and periciliary fluid surrounding the lung epithelial cells. The two next subsections present how the fluid velocity $u$ and the fluid viscosity $\eta_{G}$ are linked, and finally how the viscosity $\eta_{G}$ is modeled for such a mixture, by a Carreau law with spatially varying coefficients.

\subsection{Airway surface liquid}

The airway surface liquid (ASL) covering bronchial walls is a stratified medium composed of a mucus layer (ML) overlying a periciliary fluid layer (PCL), which behaves as a Newtonian fluid with a viscosity close to that of water. Both PCL and ML are chiefly composed of water $(>98 \%)$. Several works in the literature [25, 12, 55, 33, 39] (an exhaustive reference list is given in [56]) have considered two layers of fluid with constant fluid properties.

The periciliary fluid wetting the bronchial wall has a height generally estimated to be at least $2 / 3$ of the total ASL thickness. Once they are released by goblet cells, polymerized mucins are further hydrated, expand, and travel freely in the PCL away from the wetted surface [13, 14, 20, 4].

The innermost mucus layer results from progressive maturation and structuration of mucins in the core of the fluid. Yet, the transition between the PCL and ML layers is supposed to be continuous, assuming a progressive mucin maturation between the epithelial surface and mucus, characterized by a progressive meshwork loop size [7]. 

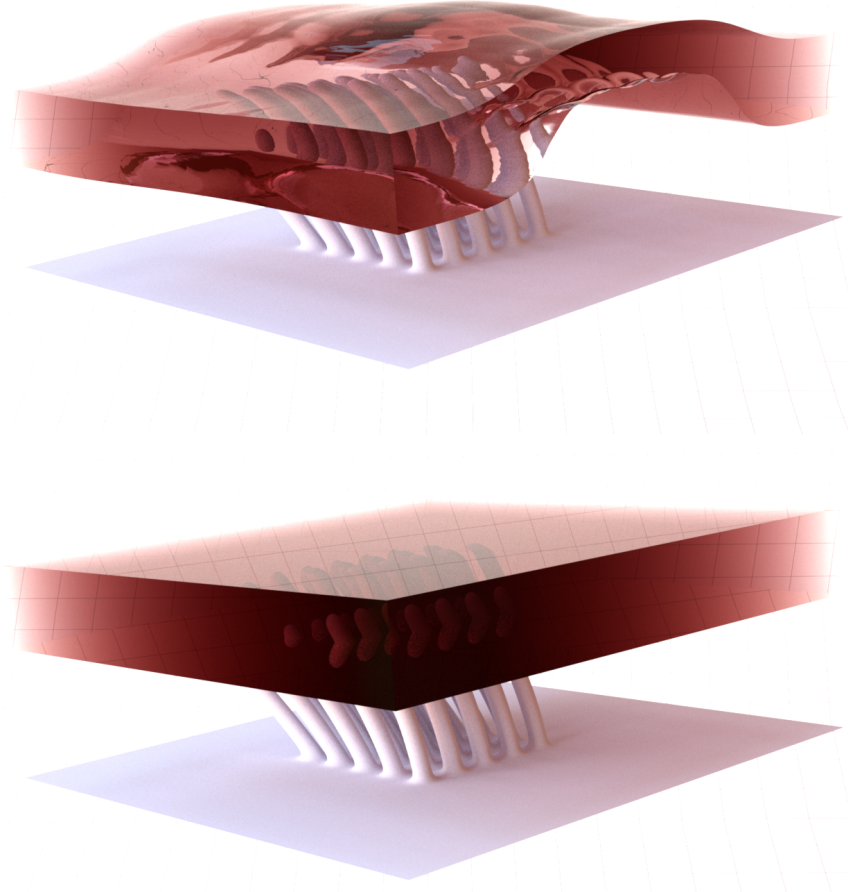

Figure 2: Evolution of isovalues of mucin maturation $\alpha \in$ $[0.4,0.9]$ (in red). Top picture: solution of transport equation (13) coupled to the 3D Stokes equation (7). Bottom picture: static mixing given by Eq. (12).

The present model considers this continuous heterogeneity. The ASL rheological parameters depends on the mucin maturation parameter $\alpha$, defined as the volumic fraction of matured mucins at a given ASL height, that is, on the cross-linking magnitude of mucin polymers [26, 48, 64]. The maturation $\alpha$ varies from 0 at the PCL-epithelium interface to 1 in the ML with a sigmoid or $\tan ^{-1}$ profile.

\subsection{Flow modeling and domain geometry}

The computational domain $\Omega$ contains a full ciliated cell (a segment of the respiratory epithelium, cf. Fig. 2). The ciliated cell is the time-dependent domain $\mathcal{B}(t)$ and the ASL domain $\mathcal{F}(t)=\Omega \backslash \overline{\mathcal{B}(t)}$. The rectangular domain is bounded by the mucus-air interface at its top and the wetted epithelial surface at its bottom, with periodical conditions in the streamwise direction.

The Reynolds, Womersley, and Froude numbers are sufficiently small to suppose a mucus regime following the stationary $3 \mathrm{D}$ Stokes equations in a time-dependent fluid domain $\mathcal{F}(t)$, that is to say the flow is always at its quasi-equilibrium [19]:

$$
-\operatorname{div}\left(2 \eta_{G} D(u)\right)+\nabla p=f \text { in } \mathcal{F}(t),
$$

where $u$ is the velocity field satisfying $\operatorname{div} u=0$ in $\mathcal{F}(t), p$ the pressure, $D(u)=\left(\nabla u+\nabla u^{T}\right) / 2$ the strain rate tensor, $\eta_{G}$ the dynamic viscosity, and $u=\bar{u}$ on boundary $\partial \mathcal{F}(t)$. The penalty

\begin{tabular}{c|c} 
Name & Value \\
\hline Cilia length & $8 \mu \mathrm{m}$ \\
Cilia diameter & $0.3 \mu \mathrm{m}$ \\
Number of Cilia & 25 \\
PCL viscosity $\left(\eta_{P C L}\right)$ & $0.001 \mathrm{~Pa} . \mathrm{s}$ \\
Viscosity transition stiffness $(\lambda)$ & 5 \\
Transition length $(\delta)$ & 0.6 \\
ASL height $(H)$ & $12 \mu \mathrm{m}$ \\
Beating frequency & $10 \mathrm{~Hz}$ \\
Inverse low-frequency cut-off $(\beta)$ & $410^{3} \mathrm{~s}$ \\
Distance between adjacent cilia & $1.5 \mu \mathrm{m}$ \\
Metachronal wavelength & $87.5 \mu \mathrm{m}$
\end{tabular}

Table 2: Parameters used for the numerical simulations, gathered from $[51,56,21,60]$ or from our own measures.

method [2] is then used to take into account the influence of the beating cilia on the fluid. It is a robust fictitious domain method compatible with any spatial discretization, in particular regular grids which permits to use fast solvers, as detailed below.

The cilia velocity $\bar{u}$ is computed from the beating model presented in [8], based on damped Young modulus propagation, and compatible with the cilia beating cycle [24, 27, 51, 40]. Since the cilia velocities are prescribed, this is a one-way fluidstructure interaction. This assumption is valid only for early stage of the sickness or for stabilized patients, that is to say for moderate deviation from the healthy case, and does not concern critical care. In particular, the modification and adaptation of the beating patterns and frequency with respect to the modification of the mucus behavior (viscosity and non-Newtonian effect) is not taken into account in this numerical model. This had been investigated experimentally [31], and the development of a coherent numerical model remains, to our knowledge, an open problem.

The set of cilia is beating, involving a sufficiently large number of cilia, conforming in-vivo [51] and in-vitro [32] cilia behavior, leading to the flow displayed on Fig. 2. The cell we consider involves 25 cilia, yielding to a sufficiently dense cilia array to get a velocity no longer depending on the number of cilia. Their configuration and simulation parameters are displayed on Table 2. Cilia beat in a coordinated manner and the resulting beating exhibits an antipleptic wave. This parameter remains constant in all the computations, its influence on the mucuciliary clearance was already investigated in the literature $[32,40,56,29,18]$.

\subsection{Rheology and maturation modeling}

As mentioned in introduction and in section 2.2, the mucus layer exhibits both shear-thinning, visco-elastic and/or viscoplastic features. While several studies have focused on the visco-elastic aspects [30, 29, 18, 55, 56, 53], visco-plastic effects are less investigated (see [41] for spatially constant Carreau law in 2D films and [12] for analytic computations). The present study focus on the shear-thinning behavior, and bring a new light on this kind of non-Newtonian feature. In order to take in account the transition between the Newtonian PCL 
and the shear-thinning mucus, an exponential growth is considered between the two fluids of viscosities $\eta_{P C L}$ and $\eta_{M L}(\dot{\gamma})=$ $K_{M L} \dot{\gamma}^{N_{M L}-1}$.

The parameters of the power-law model depend on $\alpha$ :

$$
K(\alpha)=\eta_{P C L}\left(\frac{K_{M L}}{\eta_{P C L}}\right)^{\alpha} \text { and } N(\alpha)=\alpha \cdot N_{M L}+(1-\alpha)
$$

where $\eta_{P C L}=10^{-3} \mathrm{~Pa} \cdot s$ is the Newtonian solvent viscosity assumed to the water viscosity.

The power-law model needs to be regularized in the vicinity of zero as viscosity tends to infinity. Otherwise, the effective viscosity has a lower bound at high shear rates, the solvent viscosity $\eta_{P C L}$. The Carreau model matches these two features:

$$
\eta_{G}(\alpha, u)=\eta_{P C L}+\left(\eta_{0}(\alpha)-\eta_{P C L}\right)\left[1+(\beta \dot{\gamma})^{2}\right]^{\frac{N(\alpha)-1}{2}}
$$

where the regularization parameter $\beta$ is the inverse of the cutshear rate (low shear rates; $\beta=4 \times 10^{3} s$ ). The parameter $\eta_{0}(\alpha)$ is the cut-viscosity and is determined by the choice of the power-law coefficients and cut-shear rate. This leads to:

$$
\eta_{0}(\alpha)=\eta_{P C L}\left(\frac{K_{M L}}{\eta_{P C L}}\right)^{\alpha} \beta^{\alpha\left(1-N_{M L}\right)}
$$

This class of functions is able to generate variable concentration features [57, 16].

Equation 4 corresponds to a Newtonian fluid $\eta_{G}(\dot{\gamma}, \alpha) \equiv \eta_{P C L}$ when $\alpha=0$ and evolves to the Carreau law when $\alpha$ increase to 1 in the ML:

$$
\eta_{M L}(\dot{\gamma})=\eta_{P C L}+\left(\eta_{M L}-\eta_{P C L}\right)\left[1+(\beta \dot{\gamma})^{2}\right]^{\frac{N_{M L}-1}{2}}
$$

\section{Numerical method}

Solving numerically the equation (2) in a mobile and complex geometry, could have been done using various approaches, such as accelerated kernel methods [63], immersed boundary methods designed for heterogeneous flows (See [22, 45] for its time-dependent formulation), or improved penalization techniques [2, 8, 9]. Among the different numerical methods applied to a fluid bathing a set of cilia, one can find Stokeslets methods [29, 24], finite element methods [41, 55, 56] or immersed boundary coupled to lattice Boltzmann methods [33, 39], this being not exhaustive.

In this study, we have used the equation (2) penalized by $\varepsilon^{-1}(u-\bar{u})$ over the cilia domain $\mathcal{B}(t)$, in order to satisfy the continuity between the cilia and the fluid. This is a nonlinear Stokes problem, analyzed mathematically in [10], which is robust and well-fitted to deformable geometry:

$$
-\operatorname{div}\left(2 \eta_{G}(\alpha, u) D(u)\right)+\frac{\chi(t)}{\varepsilon}(u-\bar{u})=f \text { in } \Omega,
$$

in the whole computational domain $\Omega, \bar{u}(t)$ is the cilium velocity deduced from the beating model, $\chi(t)$ the characteristic function of $\mathcal{B}(t)$, and $\varepsilon \ll 1$ the penalization parameter.

In [8], an iterative projection algorithm was developed to compute separately the pressure and the velocity field of a
Newtonian variable viscosity Stokes problem. This numerical method efficiently computes the velocity field close to the boundaries (an inherent problem of classical projection methods [28]). This original methodology allows (in the same iteration) the explicit integration of the nonlinearity of the power law so that the generalized algorithm becomes a multi-criterion fixed point.

First, we introduce a projection on divergence-free fields denoted $\Pi\left(u^{*}\right)=u^{*}-\nabla \zeta$ where $\zeta$ is the solution to the following linear elliptic partial differential equation:

$$
\left\{\begin{array}{l}
-\Delta \zeta=-\operatorname{div} u^{*} \text { in the domain } \Omega \\
\frac{\partial \zeta}{\partial n}=u^{*} \cdot n \text { on the boundary } \partial \Omega
\end{array}\right.
$$

where $n$ is the outer unit vector normal to $\partial \Omega$. This numerical algorithm relies on fast solvers [59], based on FFT decompositions, guaranteeing a quasi linear computational cost with respect to the number of grid points, which suits very well to large 3D computations.

A globally second order numerical solution of Eq. 7 is the limit of the sequence $u_{k}=\Pi\left(u_{k}^{*}\right)$ where $u_{k}^{*}$ is defined by:

$$
\left\{\begin{array}{l}
-\eta_{G}\left(\alpha, u_{k}\right) \Delta u_{k+1}^{*}+\frac{\chi(t)}{\varepsilon}\left(u_{k+1}^{*}+\Pi\left(u_{k}^{*}\right)-u_{k}^{*}-\bar{u}\right) \\
=f+\left[2 D\left(u_{k}\right)+\left(\operatorname{div} u_{k}^{*}\right) \operatorname{Id}\right] \nabla \eta_{G}\left(\alpha, u_{k}\right) \quad \text { in } \Omega \\
u_{k+1}^{*}=g-\Pi\left(u_{k}^{*}\right)+u_{k}^{*} \quad \text { on } \partial \Omega
\end{array}\right.
$$

The solution obtained by this method satisfies the boundary conditions $u=g$, which is usually not the case when such a Dirichlet conditions is set together with projection methods [28]. The equation (9) can be written

$$
-\Delta u_{k+1}^{*}+\chi(t)\left[\varepsilon \eta_{G}\right]^{-1} u_{k+1}^{*}=R H S\left(u_{k}^{*}, \bar{u}\right)
$$

where

$$
\begin{aligned}
\operatorname{RHS}\left(u_{k}^{*}, \bar{u}\right)= & \eta_{G}^{-1} f+\chi(t)\left[\varepsilon \eta_{G}\right]^{-1}\left(\bar{u}+u_{k}^{*}-\Pi\left(u_{k}^{*}\right)\right) \\
& +\left[2 D\left(u_{k}\right)+\left(\operatorname{div} u_{k}^{*}\right) \operatorname{Id}\right] \nabla \log \eta_{G}\left(\alpha, u_{k}\right)
\end{aligned}
$$

The successive iterations of equation (10) have been firstly solved by a multigrid efficient solver [1, 8]. However, this had been improved by the use of matrix perturbation theory [9]: Indeed, the penalization operator $\chi(t)\left[\varepsilon \eta_{G}\right]^{-1}$ Id in (10) exhibits large jumps, but localized, and can be seen as a large perturbation of the $-\Delta$ operator. By means of the Sherman-MorrisonWoodbury formula, this elliptic PDE is reduced to a linear system whose size is the number of grid point in the cilia body $\mathcal{B}(t)$, and whose any evaluation requires a call to a FFT solver (see [9] for more details).

The mucin maturation parameter $\alpha$ can be only a function of distance from the airway wall (called static mixing), such as the following sigmoid expression:

$$
\alpha(x, y, z)=\frac{\tan ^{-1}(\lambda(z / H-\delta))-\tan ^{-1}(-\lambda \delta)}{\tan ^{-1}(\lambda(1-\delta))-\tan ^{-1}(-\lambda \delta)}
$$

where is the ASL height is $H$, PCL height is $\delta H$, and the dimensionless transition coefficient is $\lambda$. It can also satisfy a transport 
equation (called convected mixing):

$$
\partial_{t} \alpha+u \cdot \nabla \alpha-\sigma \Delta \alpha=0
$$

where Eq. (12) then stands for the initial condition, where $\sigma$ is a numerical smoothing diffusion chosen as small as possible in order to avoid spurious jumps occurring in pure transport $\left(10^{-11} \mathrm{~m}^{2} \cdot \mathrm{s}^{-1}\right.$ for instance). When the convected mixing is used, convection is dominant and a Lagrangian method is used to integrate in time this partial differential equation [8]. Once it is coupled with the Eulerian 3D Stokes solver, it yields to a robust numerical tool, allowing large time steps without significant loss on accuracy, up to 34-fold the time step satisfying the usual transport stability condition $u_{\max } \Delta t<\Delta x$.

In the present cases, choosing static or convected mixing, whose resulting $\alpha$-isovalues are displayed on Fig. 2, leads only to a $3 \%$ difference in the mucus mean velocity. Furthermore, the case of Newtonian fluids had been investigated in [11], with linear Stokes equation. The present study focuses specifically on non-Newtonian effects on the mucociliary clearance, involving a non-linear Stokes model. The resulting non-Newtonian effects and their impact on mucociliary clearance are discussed thereafter.

\section{Phase cartography}

Measurements on mucus samples and numerical simulations enable to plot phase cartography. Resulting maps yield positions of healthy and CF mucus from the present study and literature. The effect of PCL height, which markedly impairs the mucociliary clearance, can be investigated.

The balance between PCL and ML quantified by the scalar field $\alpha$ following Eq. (12), with a PCL height is chosen as $\delta H$ where $\delta=0.6$, for a total ASL height $H=12 \mu \mathrm{m}$, representing the proximal compartment of the tracheobronchial tree, and a transition coefficient $\lambda=5$. In the second part of this study, the PCL height varies, $\delta$ ranging from 0.3 to 0.7 .

Moreover, in order to quantify the efficiency of the mucociliary clearance, the velocity is averaged both in space, over the full ML, and in time, over 5 beating cycles:

$$
\bar{U}=\frac{1}{5 T \operatorname{meas}(\Omega)} \int_{0}^{5 T} \iiint_{\Omega} u_{x}(x, y, z, t) \mathrm{d} V \mathrm{~d} t
$$

where $x$ denotes the streamwise direction given by the cilia beating.

\subsection{Cystic fibrotic mucus positioning in phase cartography}

Because the cilium beating is asymmetrical, this velocity is positive and a net displacement of the ML is observed in the direction of the esophagus. A snapshot of a simulation is presented on Fig. 2. The mean mucus velocity is computed for different values of the $\{N, K\}$ couple. Simulations were carried out for $169\{N, K\}$ couples to obtain the phase cartography of Fig. 3.

Viscosity values are not fully meaningful for shear-thinning fluids. The consistency index $K$ takes generally lower values

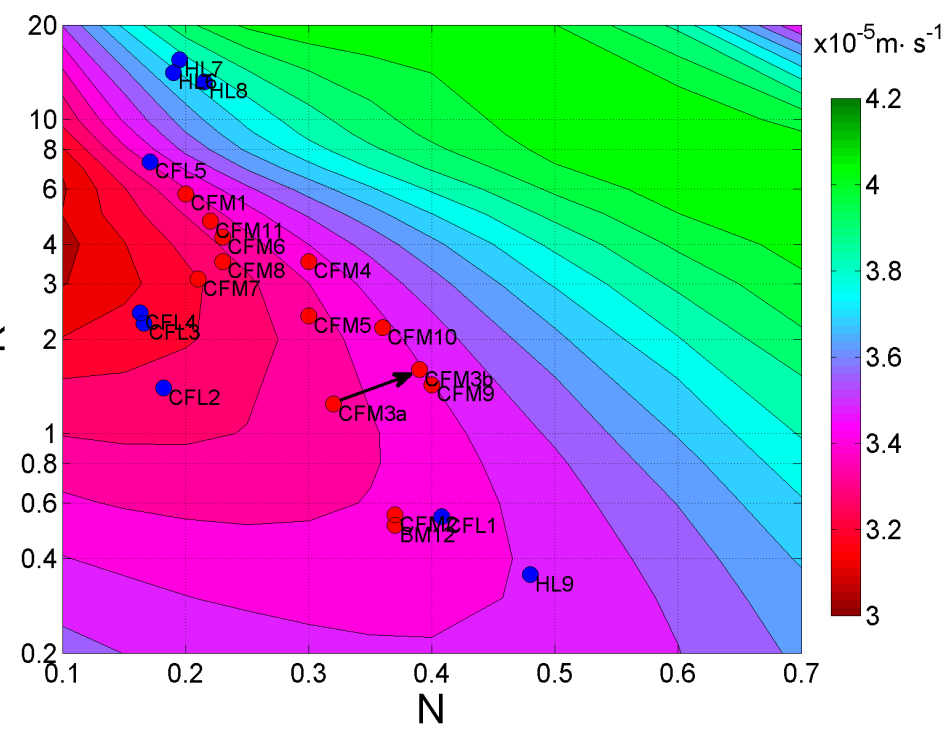

Figure 3: Phase cartography of the mucus transport velocity, with respect to rheological parameters $\{N, K\}$. Collected samples are displayed in red, while the results collected from literature are displayed in blue (Table 1).

than that in healthy subject samples. The power index varies with a greater extent in CF samples compared to values observed in healthy subject samples (except for HL9).

As depicted in Fig. 3, the mucus transport velocity does not evolve monotonously with neither $N$ nor $K$, a moderate decrease in the $K$ index leading to a lower mucus transport with a loss of velocity in the range $6-14 \%$ for our mucus samples (Table 1). An isolated drop of $6 \%$ in mucus mean velocity may suffice to be pathological. Although the HL9 datum [54] is very different from others [34, 67] (but this might be linked to a nebulization realized prior to the sampling), the mucus velocity magnitude varies less than $5 \%$ in these healthy mucus cases. The shear-thinning behavior has a strong effect, as the mucus transport velocity decays to a large extent when $\mathrm{N}$ decreases to 0.2 . This result is quite counterintuitive, but might result from a larger backflow of the mucus layer when viscosity decreases. This phenomenon depends highly on the respective ML and PCL height that affect the cilium impingement on mucus (strength and duration).

Clinically, two classes of CF mucus can be considered: stabilized and decompensated. An acute secondary infection exacerbates chronic infection with ciliated cell death, further disrupting the mucus propulsion machinery. The expectoration rheology of the CFM3 patient was measured both in stabilized and decompensated states: post-treatment sputum, after 17 days of treatment [CFM3b, stabilized] and 2 days after hospitalization [CFM3a, acute] displayed on Fig. 3 and Table 1. According to measures and computations, the mucociliary clearance improves, as expected using a combined antibiotic and steroid treatment. 
$\delta=0.7$

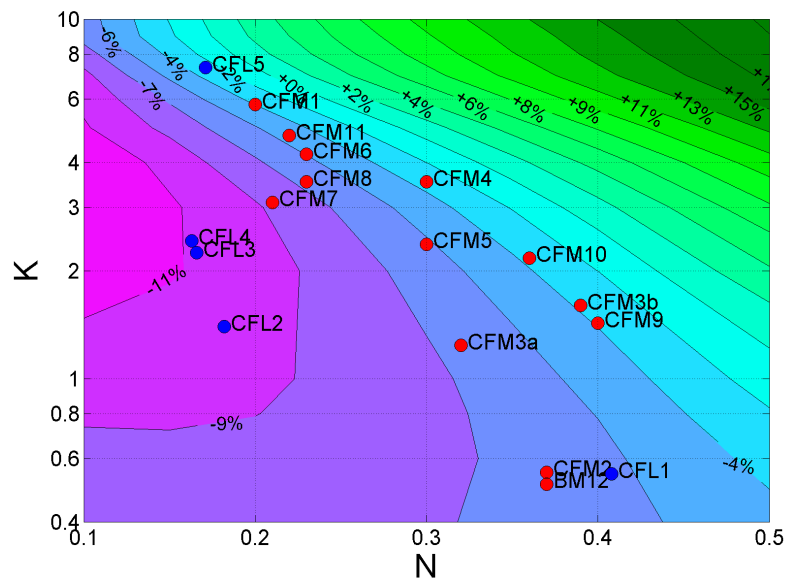

$\delta=0.5$

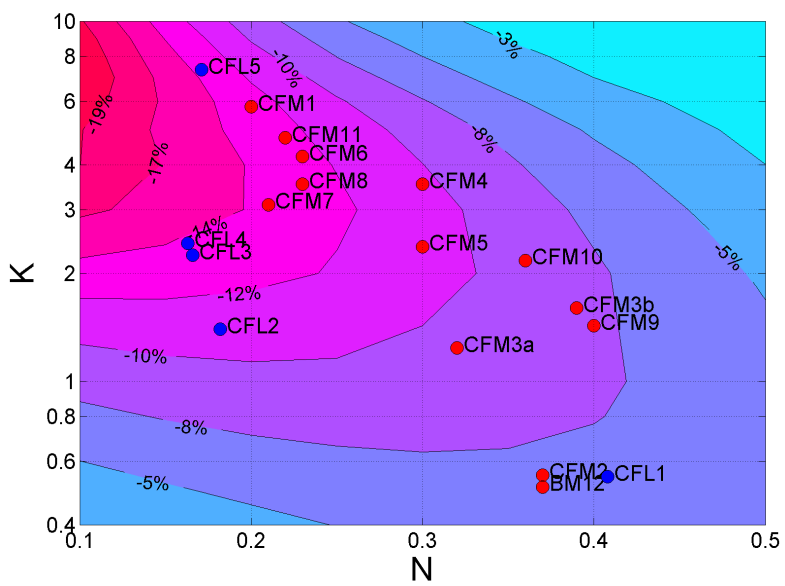

$\delta=0.3$

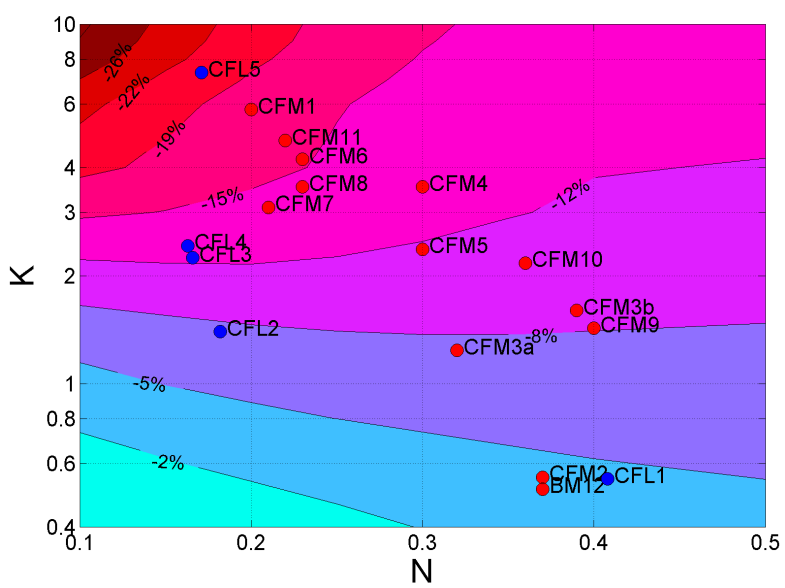

$\delta=0.6$

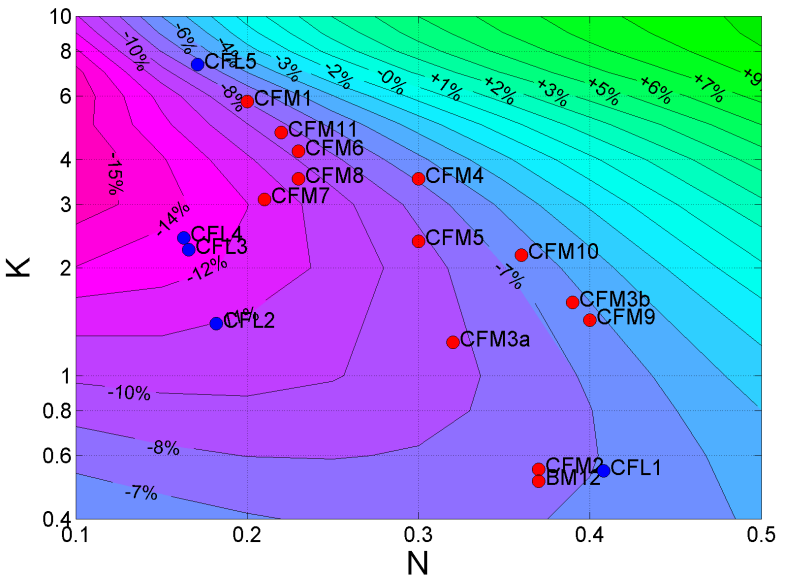

$\delta=0.4$

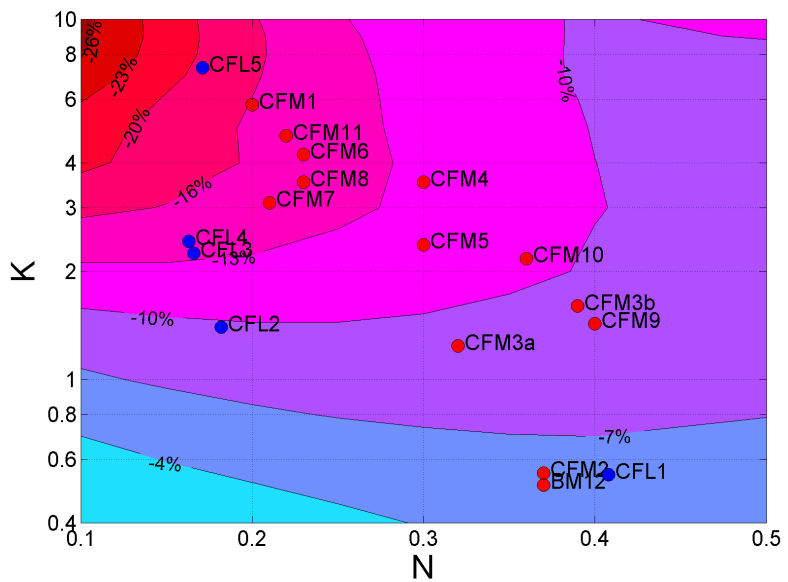

Initial profile $\alpha(z)$

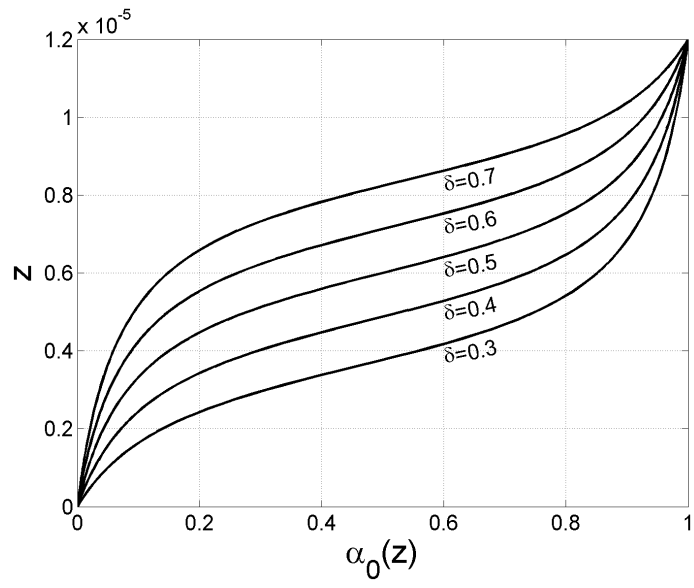

Figure 4: Loss of mucus velocity with respect to rheological parameters $(K, N)$ for several PCL/ML transition heights $\delta$, given in the bottom right plots. The reference velocity $(0 \%)$ is the healthy cases from $[34,67]$, also displayed in Fig. 3 and 1 and Table 1.

\subsection{Impact of PCL thickness}

Altered mucus velocity is shown in $\{K, N\}$ diagrams (Fig. 4) for several heights of periciliary fluid (i.e., several $\delta$ values), representing the effect of variable CFTR deficiency $[62,20]$. This alteration is given in percent of the mean healthy data (i.e.,
HL6 and HL8 in Table 1 [34, 67]).

The PCL/ML thickness ratio impacts markedly the mucus transport. The mucus velocity is reduced from $-14 \%$ to $-21 \%$ when the PCL thickness decreases ( $\delta$ falls from 0.6 to 0.3 ). Hence, for a mucus with a given set of values of the parame- 
ter couple $\{N, K\}$, a weak decrease in $\delta$ significantly reduces the mucus velocity. A limitation of this work is linked to the absence of interference between the mucus rheology and motion amplitude and frequency of respiratory cilia for a given state of ciliated cells. However, pathophysiological data are missing to handle this interaction, which can yet be explored using the present model.

\section{Conclusion}

The present work had two major objectives. Firstly, it was aimed at using an efficient solver that enables the analysis of all influent shear-thinning parameters which control the mucus motion in the respiratory tract, using a mucus maturation model closer to the biological process than a double-deck fluid domain with sharp PCF-ML transition. This mucus baths beating cilia, through which travel immersed mucin polymers that hydrate and cross-link. Secondly, it was aimed at providing a set of experimental parameters both in order to confront the numerical results (by means of healthy/pathologic classification) and to provide input rheological parameters to the numerical simulations. Despite the complexity and richness of our model, many data that are mandatory to perfectly handle the process are still missing (effect of CF on the respective thickness of the PCL and ML layers, effect of mucus viscosity on cilium beating, etc.). Nevertheless, we have shown that shear-thinning effects can of the same order as the visco-elastic effects in such environments [53].

The highly shear-thinning character of the mucus leads to very high ratio of the dynamic viscosity in the flow, around $10^{5}$ when the shear rate $\dot{\gamma}$ evolves in the range $\left[10^{-2}-10^{3}\right]$. Therefore the numerical method based on projective iteration $[8,9]$ extended herein to nonlinear viscosity proves to be extremely robust.

Cartographies show a strong influence of the power law index $N$ that must be properly incorporated in numerical simulations. In stabilized cystic fibrosis, the mucus viscosity observed for a given shear rate does not significantly differ from values observed in healthy people. Nevertheless, as the mucus velocity does not evolve monotonously with the rheological indices $N$ and $K$, mucus transport in $\mathrm{CF}$ patients can be less efficient. A vicious circle is then created, as a drop in mucuciliary clearance allow local pathogen proliferation and further deterioration of the respiratory epithelium function.

\section{Conflicts of interest declaration}

We wish to confirm that there are no known conflicts of interest associated with this publication and there has been no significant financial support for this work that could have influenced its outcome. We confirm that the article has been read and approved by all named authors. We further confirm that the order of authors listed in the paper has been approved by all of us.

\section{Acknowledgments}

This work was partially supported by the ANR Grant BioFiReaDy, under the contract number ANR-2010-JCJC0113-01. This work was also granted access to the HPC resources of P2CHPD (from Fédération Lyonnaise de Simulation et de Modélisation Numériques). We also acknowledge the use of the rheometer from the cooperative structure of joint research FERMaT (FR3089).

\section{References}

[1] Adams, J., Smolarkiewicz, P. 2001. Modified multigrid for 3D elliptic equations with cross derivatives. Applied Math. Comput. 121, 301-312.

[2] Angot, P., Bruneau, C-H., Fabrie, P. 1999. A penalization method to take into account obstacles in incompressible viscous flows. Numerische Mathematik 81(4),497-520.

[3] App, E. M., Kieselmann, R., Reinhardt, D., Lindemann, H., Dasgupta, B., King, M., Brand, P., 1998. Sputum rheology changes in cystic fibrosis lung disease following two different types of physiotherapy. Chest 114,171-177.

[4] Bansil, R. M., Stanley, E., LaMont, T. J., 1995. Mucin Biophysics. Ann. Rev. Physio. 57,635-657.

[5] Besseris, G. J., Yeates, D. B., 2007. Rotating magnetic particle microrheometry in biopolymer fluid dynamics: Mucus microrheology. J. Chem. Phys. 127, 105106.

[6] Bird, R. B., Armstrong, R. C., Hassager, O., 1987. Dynamics of Polymeric Liquids, Vol. 1, John Wiley and Sons.

[7] Button, B., Cai, L-H., Ehre, C., Kesimer, M., Hill, D. B., Sheehan, J. K., Boucher, R. C., Rubinstein, M., 2012. Periciliary brush promotes the lung health by separating the mucus layer from airway epithelia. Science 337(6097), 937-941.

[8] Chatelin, R., Poncet, P., 2013. A hybrid grid-particle method for moving bodies in 3D Stokes flow with variable viscosity. SIAM J. Sci. Comput. 35(4), B925-B949.

[9] Chatelin, R., Poncet, P., 2014. Hybrid grid-particle methods and penalization: A Sherman-Morrison-Woodbury approach to compute 3D viscous flows using FFT. J. Comput. Phys. 269, 314-328.

[10] Chatelin, R., Sanchez, D., Poncet, P., 2016. Analysis of the penalized 3D variable viscosity Stokes equations coupled to diffusion and transport. ESAIM: Math. Model. Numer. Anal. 50, 565591.

[11] Chatelin, R., Poncet, P., 2016. A parametric study of mucociliary transport by numerical simulations of 3D non-homogeneous mucus. J. Biomech. 49, 1772-1780.

[12] Craster, R. V., Matar O. K., 2000. Surfactant transport on mucus films. J. Fluid Mech. 425, 235-258.

[13] Davis, C. W., Dickey, B. F., 2008. Regulated Airway Goblet Cell Mucin Secretion. Annual Review of Physiology 70, 487-512.

[14] Davis, C. W., Lazarowski, E., 2008. Coupling of airway ciliary activity and mucin secretion to mechanical stresses by purinergic signaling. Respiratory Physiology \& Neurobiology 163, 208-213

[15] Dawson, M., Wirtz, D., Hanes, J., 2003. Enhanced viscoelasticity of human cystic fibrotic sputum correlates with increasing microheterogeneity in particle transport. J. Biol. Chem. 278(50), 50393-50401.

[16] Delgado-Reyes, V. A., Ramos-Ramirez, E. G., Cruz-Orea, A., Salazar-Montoya, J. A., 2013. Flow and Dynamic Viscoelastic Characterization of Non-Purified and Purified Mucin Dispersions. Int. J. Polymer Anal. Charact. 18, 232-245. 
[17] Derichs, N., Jin, B. J., Song, Y., Finkbeiner, W. E., Verkman, A. S., 2011. Hyperviscous airway periciliary and mucous liquid layers in cystic fibrosis measured by confocal fluorescence photobleaching. FASEB J. 25, 2325-2332.

[18] Ding, Y, Nawroth, J C, McFall-Ngai, M J, Kanso, E (2014) Mixing and transport by ciliary carpets: a numerical study. J. Fluid Mech. 743:124-140.

[19] Enault, S., Lombardi, D., Poncet, P., Thiriet, M., 2010, Mucus dynamics subject to air and wall motion. ESAIM Proc. 30, 125141.

[20] Ehre, C., Ridley, C., Thornton, D. J., 2014. Cystic fibrosis, An inherited disease affecting mucin-producing organs. Int. J. Biochem. Cell. Biol. 52, 136-145.

[21] Fahy, J. V., Dickey, B. F., 2010. Airway mucus function and dysfunction. New Engl. J. Med. 363, 2233-2247.

[22] Fai, T. G., Griffith, B. E., Mori, Y., Peskin, C. S., 2013. Immersed boundary method for variable viscosity and variable density problems using fast constant-coefficient linear solvers I: Numerical method and results. SIAM J. Sci. Comput. 35(5), B1132-B1161.

[23] Farrell, P. M., 2008. The prevalence of cystic fibrosis in the European Union. Journal of Cystic Fibrosis 7, 450-453

[24] Fauci, L. J., Dillon, R., 2006. Biofluidmechanics of Reproduction. Ann. Rev. Fluid Mech. 38, 371-394.

[25] Fulford, G., Blake, J., 1986. Muco-ciliary transport in the lung. J. Theor. Biol. 121(4), 381-402.

[26] Georgiades P, Pudney PD, Thornton DJ, Waigh T (2014) Particle tracking microrheology of purified gastrointestinal mucins. Biopolymers 101(4):366-377.

[27] Gheber L, Priel Z (1997) Extraction of cilium beat parameters by the combined application of photoelectric measurements and computer simulation. Biophys. J. 72:449-462.

[28] Guermond JL, Minev P, Shen J (2006) An overview of projection methods for incompressible flows. Comput. Methods in Appl. Mech. Engrg. 195:6011-6045.

[29] Guo, H, Nawroth, J, Ding, Y, Kanso, E (2014) Cilia beating patterns are not hydrodynamically optimal. Physics of Fluids 26:091901.

[30] Guo H, Kanso E (2016) Mucociliary Transport in Healthy and Diseased Environments. arXiv:1605.00688v2 [physics.flu-dyn].

[31] Hill, D B, Swaminathan, V, Estes, A, Cribb, J, O’Brien, E T, Davis, C W, Superfine, R (2010) Force Generation and Dynamics of Individual Cilia under External Loading. Biophysical Journal 98:57-66.

[32] Hussong J, Schorr N, Belardi J, Prucker O, Rühe J, Westerweel J (2011) Experimental investigation of the flow induced by artificial cilia. Lab. Chip 11:2017.

[33] Jayathilake PG, Tan Z, Le DV, Lee HP, Khoo BC (2012) Threedimensional numerical simulations of human pulmonary cilia in the periciliary liquid layer by the immersed boundary method. Comput. Fluids 67:130-137.

[34] Jeanneret-Grosjean A, King M, Michoud MC, Liote H, Amyot R (1988) Sampling technique and rheology of human tracheobronchial mucus. Am. Rev. Resp. Disease 137(3):707-710.

[35] Jones LC, Moussa L, Fulcher ML, Zhu Y, Hudson EJ, O’Neal WK, Randell SH, Lazarowski ER, Boucher RC, Kreda SM (2012) VAMP8 is a vesicle SNARE that regulates mucin secretion in airway goblet cells. J. Physiol. 590(3):545-561.

[36] King M (1981) Is Cystic Fibrosis Mucus Abnormal? Pediatr. Res. 15:120-122.

[37] Kocevar-Nared J, Kristl J, Smid-Korbar J (1997) Comparative rheological investigation of crude gastric mucin and natural gastric mucus. Biomaterials 18(9):677-681.
[38] Lai SK, Wang YY, Wirtz D, Hanes J (2009) Micro- and macrorheology of mucus. Adv. Drug Del. Rev. 61:86-100.

[39] Li Z, Favier J, D’Ortona U, Poncet S (2016) An immersed boundary-lattice Boltzmann method for single- and multicomponent fluid flows. J. Comput. Phys. 304, p.424-440.

[40] Mitran S (2007) Metachronal wave formation in a model of pulmonary cilia. Comput. Struct. 85:763-774.

[41] Montenegro-Johnson, T D, Smith, D J, Loghin, D (2013) Physics of rheologically enhanced propulsion: Different strokes in generalized Stokes. Physics of Fluids 25:081903.

[42] Moores C, Hudson NE, Davies A (1992) The effect of highfrequency ventilation on non-Newtonian properties of bronchial mucus. Resp. Med. 86:125-130.

[43] Nawroth J, Guo H, John D, Kanso E, McFall-Ngai M (2015) Beyond the mucus escalator: Complex ciliary hydrodynamics in disease and function. Bull. Am. Phy. Soc. 60.

[44] Norton MM, Robinson RJ, Weinstein SJ (2001) Model of ciliary clearance and the role of mucus rheology. Phys. Rev. E 83:011921.

[45] Poncet P (2009) Analysis of an immersed boundary method for three-dimensional flows in vorticity formulation. J. Comput. Phys. 228:7268-7288.

[46] Puchelle E, Zahm JM, Duvivier C, Didelon J, Jacquot J, Quemada D (1985) Elasto-thixotropic properties of bronchial mucus and polymer analogs. I: Experimental results. Biorheology 22:415-423.

[47] Puchelle E, Zahm JM, Quemada D (1987) Rheological properties controlling mucociliary frequency and respiratory mucus transport. Biorheology 24:557-563.

[48] Raynal BD, Hardingham TE, Sheehan JK, Thornton TJ (2003) Calcium-dependent protein interactions in MUC5B provide reversible cross-links in salivary mucus. J. Biol. Chem. 278:2870328710.

[49] Rubin BK, Ramirez O, Zayas JG, Finegan B, King M (1990) Collection and analysis of respiratory mucus from subjects without lung disease. Am. Rev. Respir. Dis. 141:1040-1043

[50] Sanders NN, De Smedt SC, Van Rompaey E, Simoens P, De Baets F, Demeester J (2000) Cystic fibrosis sputum: a barrier to the transport of nanospheres. Am. J. Respir. Crit. Care Med. 162(5):1905-11.

[51] Sanderson MJ, Sleigh MA (1981) Ciliary activity of cultured rabbit tracheal epithelium, Beat pattern and metachrony. J. Cell. Sci. 47:331-334

[52] Schraufnagel D (1990) Electron Microscopy of the Lung, Series Lung Biology in Health and Disease (Book 48), CRC Press.

[53] Sedaghat M H, Shahmardan M M, Norouzi M, Nazari M, Jayathilake P G (2016) On the effect of mucus rheology on the mucociliary transport. Mathematical Biosciences 272:44-53.

[54] Serisier DJ, Carroll MP, Shute JK, Young SA (2009) Macrorheology of cystic fibrosis, chronic obstructive pulmonary disease and normal sputum. Respiratory Research 10:63.

[55] Smith D, Gaffney E, Blake J (2007) A viscoelastic traction layer model of muco-ciliary transport. Bull. Math. Biol. 69(1):289327.

[56] Smith D, Gaffney E, Blake J (2008) Modelling mucociliary clearance, Respiratory Physiology \& Neurobiology 163:178-188.

[57] Soby LM, Jamieson AM, Blackwell J, Jentoft N (1990) Viscoelastic Properties of Solutions of Ovine Submaxillary Mucin. Biopolymers 29:1359-1366.

[58] Suk JS, Lai SK, Wang YY, Ensign LM, Zeitlin PL, Boyle MP, Hanes J (2009) The penetration of fresh undiluted sputum expectorated by cystic fibrosis patients by non-adhesive polymer 
nanoparticles. Biomaterials 30:2591-2597.

[59] Sweet R (1988) A Parallel and Vector Variant of the Cyclic Reduction Algorithm. SIAM J. Sci. and Stat. Comp. 9:761-766.

[60] Thiriet M (2012) Tissue Functioning and Remodeling in the Circulatory and Ventilatory Systems (Vol. 5), Series Biomathematical and Biomechanical Modeling of the Circulatory and Ventilatory Systems, Springer, New York.

[61] Tomaiuolo G, Rusciano G, Caserta S, Carciati A, Carnovale V, Abete P, Sasso A, Guido S (2014) A new method to improve the clinical evaluation of cystic fibrosis patients by mucus viscoelastic properties. PLoS ONE 9(1):e82297.

[62] Thornton DJ, Rousseau K, McGuckin MA (2007) Structure and Function of the Polymeric Mucins in Airways Mucus. Ann. Rev. Physio. 70:459-486.

[63] Tornberg AK, Greengard L (2008) A Fast Multipole Method for the Three Dimensional Stokes Equations. J. Comput. Phys. 227(3):1613-1619.

[64] Verdugo P, Aitken M, Langley L, Villalon MJ (1987) Molecular mechanism of product storage and release in mucin secretion. II. The role of extracellular Ca++. Biorheology 24:625-633.

[65] Yeates DB, Besseris GJ, Wong LB (1997) Physicochemical properties of mucus and its propulsion. The Lung : Scientific foundations, second edition, eds. Crystal RG, West JB et al., LippincottRaven Publishers, Philadelphia.

[66] Zamankhan P, Helenbrook BT, Takayama S, Grotberg JB (2012) Steady motion of Bingham liquid plugs in two-dimensional channels. J. Fluid Mech. 705:258-279.

[67] Zayas JG, Man GCW, King M (1990) Tracheal mucus rheology in patients undergoing diagnostic bronchoscopy. Interrelations with smoking and cancer. Am. Rev. Respir. Dis. 141:1107. 